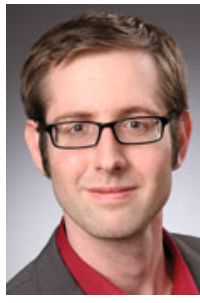

Daniel Pfeiffer

Jahrgang 1982. 2002-2009

Diplomstudium der Technischen Biologie an der Universität Stuttgart. 2009-2013 Promotion am Institut für Mikrobiologie der Universität Stuttgart als Stipendiat der Landesgraduiertenförderung in der Arbeitsgruppe von

Prof. Dr. D. Jendrossek. Seit 2014 wissenschaftlicher Mitarbeiter am Lehrstuhl für Mikrobiologie der Universität Bayreuth.

DOI: $10.1007 / \mathrm{s} 12268-015-0546-2$

(C) Springer-Verlag 2015

Viele Mikroorganismen bilden Polyhydroxyfettsäuren als Speicherstoffe für Energie und Kohlenstoff. Hierbei handelt es sich um aliphatische Polyester; aufgrund ihrer Materialeigenschaften sind diese als Biokunststoffe von besonderem Interesse. Am weitesten verbreitet ist die Poly(3-hydroxybuttersäure) (PHB). In der Zelle liegt PHB in wasserunlöslicher granulärer Form vor. PHBGranula sind dabei von einer Hüllschicht aus Proteinen umgeben. An der Oberfläche der Granula sind Proteine gebunden, die für deren Bildung (PHB-Synthase, PhaC) oder Abbau (PHB-Depolymerasen, PhaZ) verantwortlich sind bzw. die Größe und Anzahl der Granula regulieren (Phasine, PhaP).

Ein Ansatz zur Auffindung neuartiger PHBgebundener Proteine über Proteinwechselwirkungen führte zur Entdeckung bisher unbekannter Proteine im Modellorganismus der PHB-Bildung, Ralstonia eutropha [1]. Dieses Gram-negative Bodenbakterium ist wegen seiner Fähigkeit, PHB bis zu 90 Prozent seines Zelltrockengewichts speichern zu können, auch für die industrielle Produktion von PHB von Bedeutung. Hierbei wurde das Protein PhaM als Interaktionspartner der PHB-Synthase identifiziert (Abb. 1A). PhaM

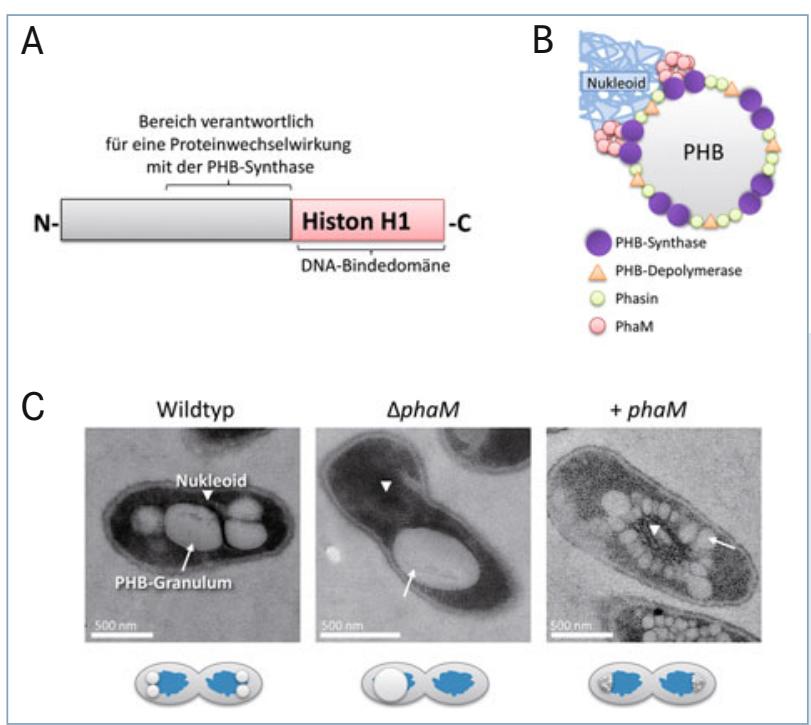

VAAM-Promotionspreis 2014

\title{
PHB als bakterieller Kohlenstoffspeicher ist am Nukleoid verankert
}

\author{
DANIEL PFEIFFER \\ LEHRSTUHL FÜR MIKROBIOLOGIE, UNIVERSITÄT BAYREUTH
}

beeinflusst neben der Größe und Anzahl gebildeter PHB-Granula deren Lokalisierung in der Zelle.

Fluoreszenz- und elektronenmikroskopische Untersuchungen zeigten, dass PhaM in der Lage ist, PHB-Granula über eine Histonähnliche DNA-Bindedomäne an das Nukleoid (Bakterienchromosom) zu binden [1, 2]. Hierdurch wird gewährleistet, dass PHB-Granula im Zuge der Zellteilung gleichmäßig auf beide Tochterzellen verteilt werden (Abb. 1C, links). Zellen ohne phaM-Gen sind hierin beeinträchtigt und bilden zudem meist nur ein bis zwei sehr große PHB-Granula (Abb. 1C, Mitte). Bei einer künstlich herbeigeführten Überproduktion von PhaM kommt es hingegen zur Bildung vieler kleiner Nukleoid-gebundener PHB-Granula (Abb. 1C, rechts). Wir konnten zeigen, dass dieser Effekt u. a. auf einer Erhöhung der enzymatischen Aktivität der PHB-Synthase durch PhaM beruht [3]. Die PHB-Synthase katalysiert den letzten Schritt der Biosynthese von PHB, indem sie mit Koenzym A aktivierte Einheiten der $(R)$-3-Hydroxybuttersäure zum PHB verknüpft. Ein hochmolekularer Nukleoidgebundener Proteinkomplex aus PHB-Synthase und PhaM stellt vermutlich den Ausgangspunkt für die Bildung neuer PHB-Granula dar (Abb. 1B). Somit wird gewährleistet, dass neu gebildete PHB-Granula direkt mit dem Nukleoid verknüpft und später gleichmäßig auf beide Tochterzellen verteilt werden.
Diese Ergebnisse zeigen, dass Bildungsort und intrazelluläre Lokalisierung von PHBGranula genau reguliert werden. Nach heutigem Wissensstand sollten PHB-Granula deshalb eher als komplexe subzelluläre organellenartige Strukturen (Carbonosomen) und nicht nur als einfache Polymerspeicher betrachtet werden.

\section{Danksagung}

Herzlicher Dank geht an Prof. Dr. Dieter Jendrossek, in dessen Arbeitsgruppe die Arbeiten durchgeführt wurden. Zudem bedanke ich mich bei allen anderen Mitarbeitern des Instituts für Mikrobiologie der Universität Stuttgart. Ferner danke ich der Landesgraduiertenförderung und der Deutschen Forschungsgemeinschaft für ihre Unterstützung.

\section{Literatur}

[1] Pfeiffer D, Wahl A, Jendrossek D (2011) Identification of a multifunctional protein, PhaM, that determines number, surface to volume ratio, subcellular localization and distribution to daughter cells of poly(3-hydroxybutyrate), PHB, granules in Ralstonia eutropha H16. Mol Microbiol 82:936-951 [2] Wahl A, Schuth N, Pfeiffer D et al. (2012) PHB granules are attached to the nucleoid via PhaM in Ralstonia eutropha. BMC Microbiol 12:262

[3] Pfeiffer D, Jendrossek D (2014) PhaM is the physiological activator of PHB synthase (PhaC1) in Ralstonia eutropha. Appl Environ Microbiol 80:555-563
Korrespondenzadresse:
Dr. Daniel Pfeiffer
Lehrstuhl für Mikrobiologie
Universität Bayreuth
Gebäude NW I
Universitätsstraße 30
D-95447 Bayreuth
Tel.: 0921-55-2595
daniel.pfeiffer@uni-bayreuth.de

4 Abb. 1: Der bakterielle Kohlenstoffspeicher Poly(3-hydroxybuttersäure) (PHB) in Ralstonia eutropha. A, Das 27-Kilodalton-Protein PhaM verknüpft PHB-Granula mit dem Nukleoid über seine C-terminale DNA-Bindedomäne. Der N-terminale Teil von PhaM geht eine Proteinwechselwirkung mit der PHB-Synthase ein. B, Ein Nukleoid-gebundener Proteinkomplex aus PHB-Synthase (violett) und PhaM (rosa) stellt den Ausgangspunkt für die Bildung neuer Granula dar. An die Granula binden weitere Proteine, wie Phasine (grün) oder PHB-Depolymerasen (orange). C, elektronenmikroskopische Aufnahmen von Ralstonia eutropha-Wildtyp (links), phaM-Deletionsmutante (Mitte) und phaM-Überexpressionsstamm (rechts). 